\title{
Comparación del Desarrollo Embrionario de Piaractus brachypomus (Serrasalmidae) y Pseudoplatystoma sp. (Pimelodidae)
}

\author{
Comparison of the Embryonic Development of Piaractus brachypomus (Serrasalmidae) and \\ Pseudoplatystoma sp. (Pimelodidae)
}

***,**** John Díaz Olarte; ${ }^{*, * * * *}$ Lili J. Marciales-Caro; ${ }^{* * * * * *}$ Freddy Cristancho Vásquez; ${ }^{* * *}$ Pablo E. Cruz-Casallas

DÍAZ-OLARTE, J.; MARCIALES-CARO L. J.; CRISTANCHO, V. F. \& CRUZ-CASALLAS, P. E. Comparación del desarrollo embrionario de Piaractus brachypomus (Serrasalmidae) y Pseudoplatystoma sp. (Pimelodidae). Int. J. Morphol., 28(4):1193-1204, 2010.

RESUMEN: Pseudoplatystoma sp y Piaractus brachypomus son dos especies de peces dulceacuícolas endémicas de América del Sur de alto interés comercial como peces de cultivo. El objetivo de este trabajo fue comparar el desarrollo embrionario hasta la eclosión en estas dos especies. Para observar el desarrollo embrionario se incubaron oocitos fertilizados, obtenidos por inducción hormonal, en incubadoras de flujo ascendente tipo Woynarovich bajo condiciones controladas de calidad de agua. Los clivajes, la fase de blástula, gástrula hasta el cierre del blastoporo y eclosión en Pseudoplatystoma sp se observaron entre las 0:30 - 18:00 HPF, mientras que en P. brachypomus fue más rápida, observándose desde las 0:30 hasta las 14:00 HPF, siendo más homogénea que en Pseudoplatystoma sp.

PALABRAS CLAVE: Desarrollo embrionario; P. Brachypomus; Pseudoplatystoma sp.

\section{INTRODUCCIÓN}

La cachama blanca (Piaractus brachypomus) y el bagre rayado (Pseudoplatystoma $s p$ ) son dos especies de peces endémicas de Suramérica (Fabré et al., 2000; Landines \& Mojica, 2006), las cuales actualmente forman parte de los sistemas de producción piscícola. La mayoría de silúridos y algunos carácidos presentan elevadas tasas de mortalidad durante la etapa de larvicultura y alevinaje, debido principalmente a depredación intra-específica, conocida también como canibalismo fraternal o intracohorte (Kossowski, 1996; Qin \& Fast, 1996; Segura et al., 2004; Nuñez et al., 2008), el cual es favorecido por las diferencias en el tiempo de eclosión, la heterogeneidad en las tasas de crecimiento, la inadecuada alimentación y la utilización de densidades de alojamiento inapropiadas (Kossowski; Atencio-García \& Zaniboni-Filho, 2006).

Las observaciones del desarrollo embrionario en silúridos muestran que el periodo hasta la eclosión es más largo que en otras especies, tales como las de carácidos, lo cual se puede atribuir en parte a la presencia de una doble membrana, que consiste en una capa gelatinosa externa y un gran espacio perivitelínico, característica común a las especies neotropicales reofílicas o que presentan migración reproductiva (Atencio-García \& Zaniboni-Filho; Cardoso et al., 1995). Esta doble membrana funciona como una adaptación morfológica que brinda protección al embrión en aguas con alta turbiedad (Cardoso et al.; Pereira et al., 2006). Las diferencias en la duración del periodo desde la fertilización hasta la eclosión, produce heterogeneidad en la talla de las larvas y en la relación tamaño de la boca/longitud total, lo cual favorece notablemente el desarrollo de conductas caníbales durante la etapa larval.

De acuerdo con lo anterior, el estudio del desarrollo embrionario y larvario de estas especies permitiría ajustar técnicas de manejo más adecuadas durante las fases de incubación, larvicultura y alevinaje (Zaniboni-Filho et al., 2001). Por lo tanto, el objetivo de este estudio fue examinar

* Maestría en Estadística, Facultad de Ciencias, Universidad Nacional de Colombia, Bogotá, Colombia.

** Instituto de Acuicultura, Facultad de Ciencias Agropecuarias y Recursos Naturales, Universidad de los Llanos, Villaviencio, Meta, Colombia.

**** Maestría en Ciencias Biológicas, Facultad de Ciencias, Universidad de los Andes, Bogotá, Colombia.

***** Escuela de Biología, Facultad de Ciencias, Universidad Industrial de Santander, Bucaramanga, Santander, Colombia. 
y comparar los estados iníciales del desarrollo embrionario entre Piaractus brachypomus y Pseudoplatystoma sp, bajo condiciones controladas de laboratorio.

\section{MATERIAL Y MÉTODO}

Las larvas de bagre rayado (Pseudoplatystoma $s p$ ) y cachama ( $P$. brachypomus) fueron obtenidas por reproducción inducida hormonalmente en el instituto de Acuicultura (IALL) de la Universidad de los Llanos (UNILLANOS), localizado en el kilometro 12 vía Puerto López, vereda Barcelona del municipio de Villavicencio, departamento del Meta, a 418 m.s.n.m. Se utilizaron reproductores mantenidos en cautiverio, los cuales fueron tratados con hormonas para inducir la maduración final de las gónadas, la ovulación y espermiación. Para este propósito fue utilizado Extracto de hipófisis de carpa (EHC), siguiendo los protocolos establecidos para cada una de las especies, así: Para las hembras de $P$. brachypomus, dos inyecciones de $0.5 \mathrm{mg} . \mathrm{kg}-1$ y 5 mg.kg-1 vía Intramuscular (IM), administradas con 12 h de intervalo (Landines \& Mojica) y para las hembras de Pseudoplatystoma sp, tres inyecciones de $0.25 \mathrm{mg} . \mathrm{kg}-1,0.5$ mg.kg-1 y 5 mg.kg-1, administradas vía IM, con 24 y 12 h de intervalo, respectivamente (Mira et al., 2007). La espermiación en las dos especies se estimuló aplicando una única dosis de 4 mg.kg-1 de EHC, aplicada vía IM, simultáneamente con la última inyección de las hembras.

Los oocitos y el semen fueron obtenidos por extrusión y el proceso de fertilización se realizó en seco. Los oocitos seminados fueron llevados a incubadoras Woynarovich de $200 \mathrm{~L}$ de capacidad, manteniendo un flujo ascendente continuo de 6 - 8 L.min-1. Tanto para P. brachypomus como para Pseudoplatystoma sp, durante el desarrollo embrionario se tomaron muestras al azar (c.a. 100 oocitos fertilizados), así: cada 15 min desde la fertilización hasta tres horas post fertilización (HPF) y cada hora, desde las tres HPF hasta la eclosión. Las muestras fueron preservadas en solución del $10 \%$ de formalina buferada. En el momento de cada muestreo, se midieron los parámetros físicos y químicos del agua, tales como temperatura $\left({ }^{\circ} \mathrm{C}\right)$, oxígeno disuelto (mg. L-1), $\mathrm{pH}$, sólidos disueltos totales TDS (mg.L-1) y conductividad ( $\mu \mathrm{s} . \mathrm{cm}-1)$, usando una sonda multiparamétrica (YSI Professional Plus, USA).

Las muestras fueron observadas y descritas con la ayuda de un microscopio óptico (Carl Zeiss) y un estereomicroscopio (Carl Zeiss), adicionalmente se tomaron registros fotográficos. Para cada una de las muestras se realizaron recuentos de las estructuras observadas, para establecer los porcentajes relativos de los diferentes estadios del desarrollo embrionario a lo largo del tiempo. La terminología utilizada para describir los diferentes estadios del desarrollo embrionario se basó en los trabajos de Godinho et al. (1978); Cussac et al. (1985); Cardoso et al.; Botero et al. (2004); Faustino et al. (2007); Pereira et al.; NinhausSilveira et al. (2006); Machinandiarena et al. (2003) y Marques et al. (2008).

\section{RESULTADOS}

\section{Desarrollo Embrionario de Pseudoplatystoma sp y $P$. brachypomus.}

La Tabla I resume las características físicas y químicas del agua en las incubadores Woynarovich durante el desarrollo embrionario de Pseudoplatystoma sp y $P$. brachypoтus, las cuales se mantuvieron relativamente constantes y corresponden a los rangos de confort determinados para las especies estudiadas (Núñez et al.; Landinez \& Mojica).

Los principales eventos y características en la secuencia del desarrollo embrionario de cada especie, desde las 0:15 horas post fertilización (HPF) hasta la eclosión, se resumen en la Tabla II.

Oocitos. Los oocitos de Pseudoplatystoma sp al momento del desove presentan un diámetro de $850-900 \mu \mathrm{m}$ y una coloración amarillenta, mientras que los de $P$. brachypomus presentaron 1000 - $1200 \mu \mathrm{m}$ de diámetro y de color verdeazulado; Las dos especies se caracterizan por tener abundante vitelo (telolecitos). Después de fertilizados aumentan de tamaño debido a la hidratación y, en consecuencia, los oocitos hidratados de Pseudoplatystoma sp alcanzaron un diámetro de $1930 \pm 40 \mu \mathrm{m}$, mientras que los de $P$. brachypoтиs $1680 \pm 34 \mu \mathrm{m}$.

Cigoto. Este periodo comprende desde la fertilización hasta el primer clivaje. En Pseudoplatystoma sp y P. brachypomus se observó que el citoplasma toma una coloración más oscura debido a la segregación citoplasmática. En las dos especies, hacia las 0:15 horas post fertilización, ocurrió la diferenciación entre el polo animal y vegetal, evidenciado el primero por la mayor concentración de citoplasma (blastodisco) y el segundo por la concentración de vitelo (Fig. 1-a; 2-a).

Clivajes. El clivaje para ambas especies fue meroblástico. De esta forma, el primer clivaje ocurrió para ambas especies a partir de las 0:30 HPF (Fig. 1b, 2b-c), dividiéndose en un plano meridional para formar dos células de igual tama- 

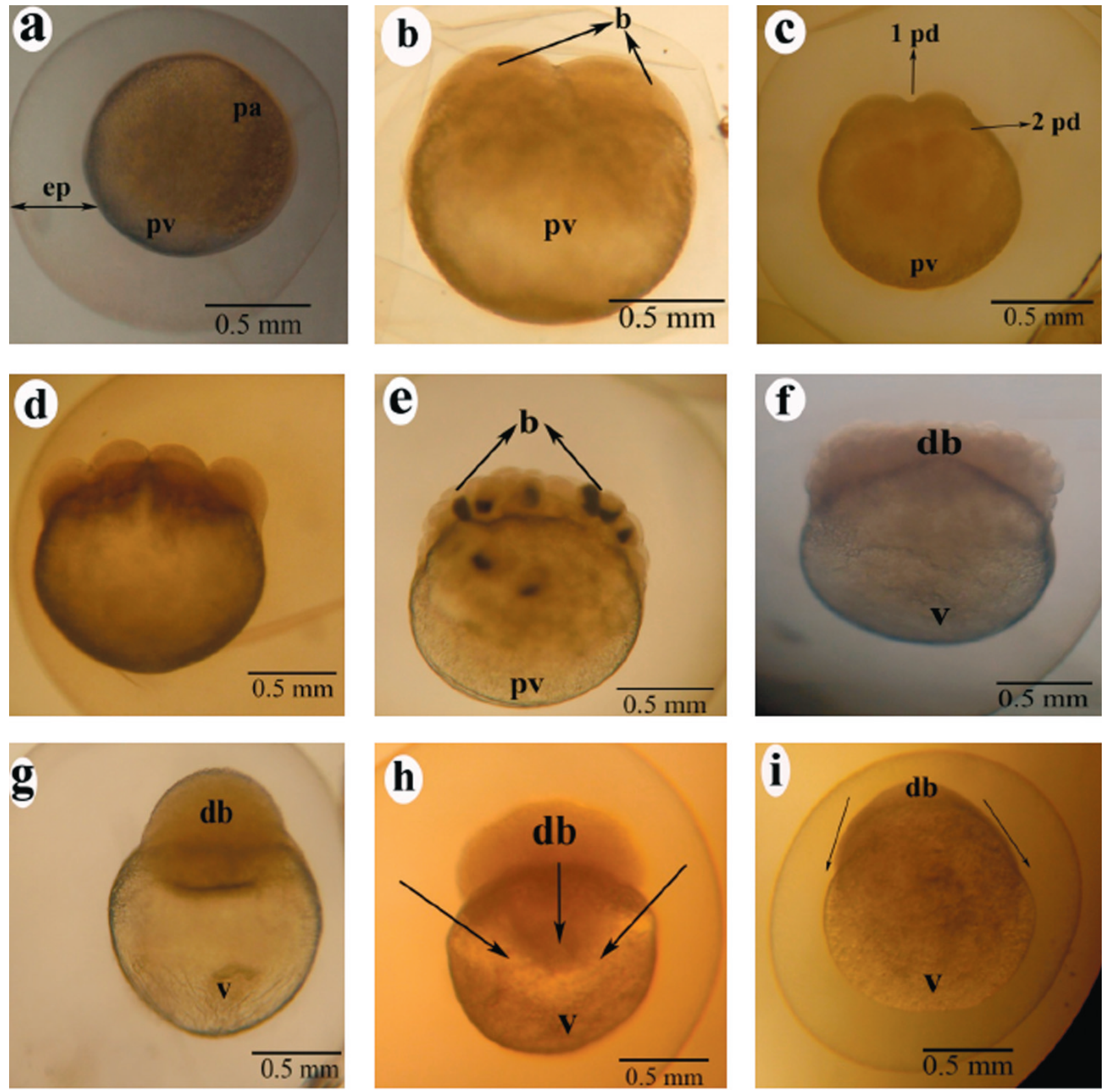

Fig. 1. Desarrollo embrionario de Pseudoplatystoma sp (Bagre rayado). a) blastodisco unicelular, b) estado de 2 células, c) estado de 4 células, d) estado de 8 células, e) estado 64-128 células, f) blastodisco inicial, g) elevación del blastodisco, h-i) inicio descenso del blastodisco sobre el polo vegetal. (pa) polo animal, (pv) polo vegetal, (ep) espacio perivitelinico, (b) blastómero, (pd) plano de división, (db) discoblástula, (v) vitelo.

Tabla I. Características físicas y químicas del agua de las incubadoras de flujo ascendente tipo Woynarovich, durante el desarrollo embrionario de Piaractus brachypomus y Pseudoplatystoma sp hasta su eclosión.

\begin{tabular}{|c|c|c|c|c|c|c|c|}
\hline & $\begin{array}{l}\text { Temperatura } \\
\left({ }^{\circ} \mathrm{C}\right)\end{array}$ & $\begin{array}{l}\text { Oxígeno } \\
\text { mg.L-1 }\end{array}$ & Disuelto & $\mathrm{pH}$ & $\begin{array}{l}\text { Salinidad } \\
(\mathrm{ppm})\end{array}$ & $\begin{array}{l}\text { Conductividad } \\
(\mathrm{mS} . \mathrm{cm}-1)\end{array}$ & $\begin{array}{l}\text { Total de Sólidos } \\
\text { Disueltos }\end{array}$ \\
\hline P. brachypoтиs & $26.9 \pm 0.4$ & $3.9 \pm 0.4$ & & $6.7 \pm 0.3$ & $0.02 \pm 0$ & $52 \pm 2$ & $32.8 \pm 0.4$ \\
\hline Pseudoplatystoma $s p$ & $27.1 \pm 0.5$ & $4.1 \pm 0.8$ & & $6.4 \pm 0.4$ & $0.01 \pm 0$ & $48 \pm 2$ & $41.8 \pm 0.1$ \\
\hline
\end{tabular}



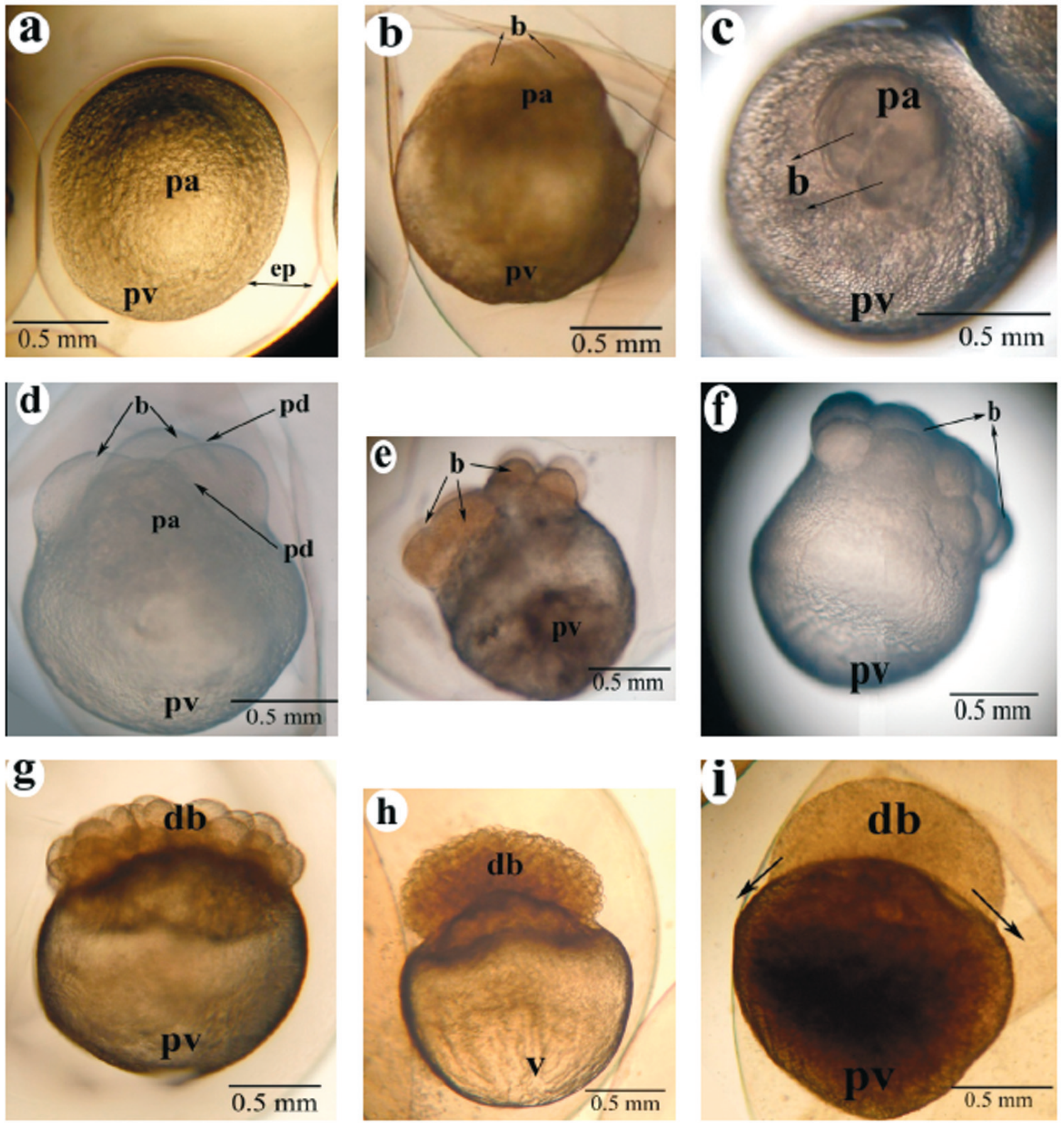

Fig. 2. Desarrollo embrionario de Piaractus brachypomus (Cachama). a) blastodisco unicelular, b-c) estado de 2 células, d) estado de 4 células, e) estado 8 células, f) estado 16 células, g) estado 64 células, h) blastodisco inicial, i) inicio descenso del blastodisco sobre el polo vegetal. (pa) polo animal, (pv) polo vegetal, (ep) espacio perivitelinico, (b) blastómero, (pd) plano de división, (db) discoblastula, (v) vitelo.

ño. La siguiente división se observó para ambas especies entre las 0:45 - 1:00 HPF (Fig. 1c, 2d); esta división ocurrió perpendicular a la primera, originando cuatro células de igual tamaño (blastómeros). Entre las 0:45-1:00 HPF en Pseudoplatystoma sp y 0:45-1:15 HPF en $P$. brachypomus, ocurrió el tercer clivaje, originando dos filas de cuatro blastómeros cada una, producto de una doble división paralela al primer eje de clivaje (Fig. 1d, 2e). El cuarto clivaje en Pseudoplatystoma sp se observó entre 1:00 - 1:15 HPF, en cambio para $P$. brachypomus se observó entre 1:00 -1:30 HPF (Fig. 2f); esta división ocurrió paralela al segundo plano de clivaje, generando cuatro filas de cuatro blastómeros. 
Tabla II. Cronología y descripción de los eventos principales del desarrollo embrionario de cachama blanca y (P. brachypomus) y bagre rayado (Pseudoplatystoma sp).

Tiempo post Características observadas

fertilización

Piaractus brachypomus

Pseudoplatystoma sp.

$\begin{array}{lll}0: 15 \mathrm{~h} & \begin{array}{l}\text { Periodo de cigoto, se diferencia claramente el polo } \\ \text { animal y el polo vegetal (Fig. 2a). }\end{array} & \begin{array}{l}\text { Se diferenc } \\ \text { (Fig. 1a) }\end{array}\end{array}$

0:30 h Primer clivaje originando 2 células (Fig. 2 b-c). Primer clivaje originando 2 células (Fig.1b).

0:45 h Segundo clivaje originando 4 células (Fig. 2d). Segundo clivaje originando 4 células (Fig. 1c).

1:00 h Tercera división formando 8 células (28\%) (Fig. 2e) y cuarta división 32 (33\%) (Fig. 2f).

Se observan zigotos en tercera división ( 8 células) (33\%)

(Fig.1d), cuarto clivaje (16 células en arreglo $4 \times 4)(32 \%)$

(Fig. 1c), quinto y sexto clivaje (20\% y $12 \%)$.

1:15 h Cuarto plano de segmentación, estado de 16 células (arreglo 4 x 4) (Fig. 2f).

Se observan zigotos en estado de 32 células (quinto clivaje en arreglo $8 \times$ 4) (Fig. 1e) y 64 células (sexto clivaje blastómeros muy pequeños).

1:30 h Estado de 32 células (Fig. 2g).

Complejo multicelular formando el blastodisco (blastodisco inicial) (Fig. 1f). Igualmente se observan zigotos de 32 y 64 células.

1:45 h Estado de 64 células e inicio de blástula.

2:00 h Inicio de blástula (moña). (Fig. 2h)

3:00 h Blástula inicial (86\%) yel evación del blastodisco (14\%). (Fig. 2i), (Fig. 4a).

4:00 h Anillogerminal.

5:00 h Periodo de gástrula 50\% (Fig. 4a).

7:00 h $\quad 80 \%$ de epibólia en gastrulación (Fig. 4b).

8:00 h Cubre totalmente el saco vitelino. Se observa el cierre del blastóporo.

10:00 h Periodo de segmentación (Fig. 4c-d).

12:00 h Presencia de somitas y saco vitelino.

13:00 h Periodo de segmentación. Eclosión.
Se observa zigotos con un blastodisco inicial y o tros con un blastodisco más segmentado (Fig. 1g).

Se observa zigotos en 3 es tados de blastulación: inicial (Fig. 1h), blastodisco más segmentado (Fig. 1g) y elevado (Fig. 1 h).

Se observa zigotos en 2 estados: Blastodisco más segmentado y empieza a descender dando inicio de la gastrulación (Fig. 1i).

Una parte de los zigotos el blastodisco desciende. Otra parte inicia los movimientos epibólicos (Gástrula inicial): las células blastódermicas y periblásticas recubren el saco vitelino (Fig 3a).

Epibólia del $80 \%$, se observa la mitad del vitelo recubierto por las células periblásticas (Gástrula intermedia) (Fig. 3b).

Recubre el saco vitelino en un 90\% (Gastrulación avanzada).

Ocurre el cierre del blastóporo (Fig. 3c)

Se observa un embrión diferenciado cráneo-caudal, dispuesto sobre el saco de la yema en forma arqueada. Se observa las primeras somitas (Fig. 3 d-e).

Se observan 12-14 pares de somitas.

Movimientos autóctonos fuertes y continuos girando sobre su propio eje. Eclosión de algunos embriones (Fig. 3f). 

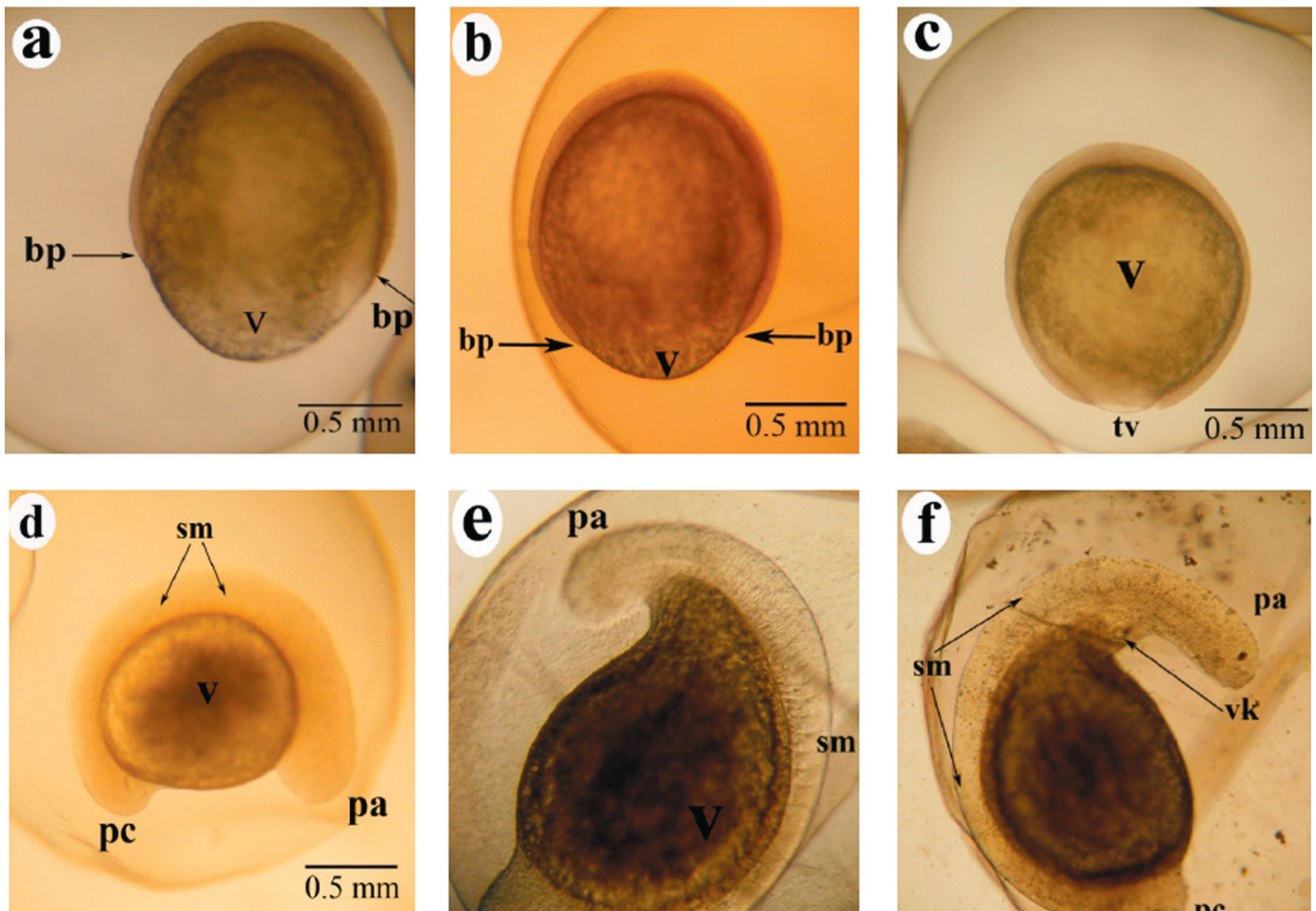

Fig. 3. Formación de la gástrula y organogénesis en Pseudoplatystoma sp. a)
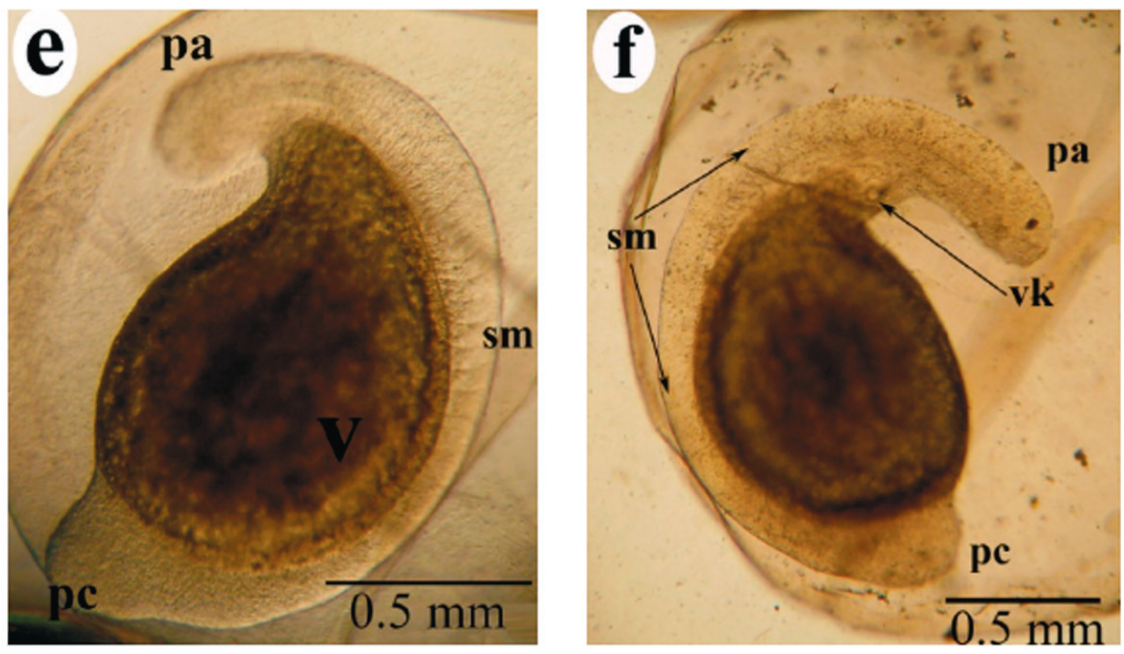
epibólia del 80\%, b) epibólia del $95 \%$, c) cierre del blastoporo, d-f) embrión diferenciado antero-posterior en forma arqueada. (bp) borde periblasto, (v) vitelo, (tv) tapón vitelino, (sm) somitas, (vk) vesícula de Kupffer, (pa) parte caudal, (pc) parte cefálica.

Del quinto y sexto clivajes se originan 32 y 64 blastómeros, de los cuales es difícil establecer los planos de división, en virtud del tamaño y disposición de las células; estos ocurrieron entre 1:00 - 2:00 HPF en Pseudoplatystoma sp y entre 1:00 -1:45 HPF en P. brachypomus.

Blástula. La blástula se observó como una capa celular que empieza a envolver el vitelo, la cual recibe la denominación de blastodisco, dándole al embrión una forma ovalada. Este estado fue diferenciado en tres etapas: etapa inicial, etapa de elevación de la blástula y etapa de descenso; esta última marca el inicio de la gastrulación por los movimientos morfogenéticos de la epibolia. En Pseudoplatystoma sp se observó desde la etapa inicial hasta el descenso o inicio de la gastrulación desde la 2:00 - 5:00 HPF (Fig. 1f-i), mientras que en P. brachypomus se observó entre la 2:00 - 4:00 HPF (Fig. 2h-i).

Gástrula. Se observaron cuatro etapas, una inicial cuando se dio el movimiento progresivo de células hacia el polo vegetal (epibolia), la siguiente cuando la epibolia llegó al 50\%, luego cuando el porcentaje de epibolia llegó al 80-95\% (se observa el tapón vitelino) y en la última etapa se observó el cierre del blastoporo, haciéndose más evidente la diferenciación del eje cráneo-caudal (Fig. 3a-c; 4a-b). En Pseudoplatystoma sp el estado de gástrula se observó desde la 5:00 - 10:00 HPF, mientras que en P. brachypomus se observó desde la 5:00 - 8:00 HPF, siendo más corta esta etapa para esta especie.

Organogénesis. En esta etapa se observó un embrión con una diferenciación céfalo- caudal, con simetría bilateral, dispuesto sobre el vitelo tomando una forma arqueada inicial, para finalmente cambiar a una forma totalmente extendida hasta su eclosión. Según como se hacían visibles las estructuras se clasificó en cuatro fases: una fase inicial (organogénesis i) que comprende la diferenciación de órganos cefálicos como la vesícula óptica y ótica; una segunda fase (organogénesis ii) donde se diferenció la parte caudal (cola). En la tercera fase (organogénesis iii) se diferenciaron las cavidades encefálicas, 

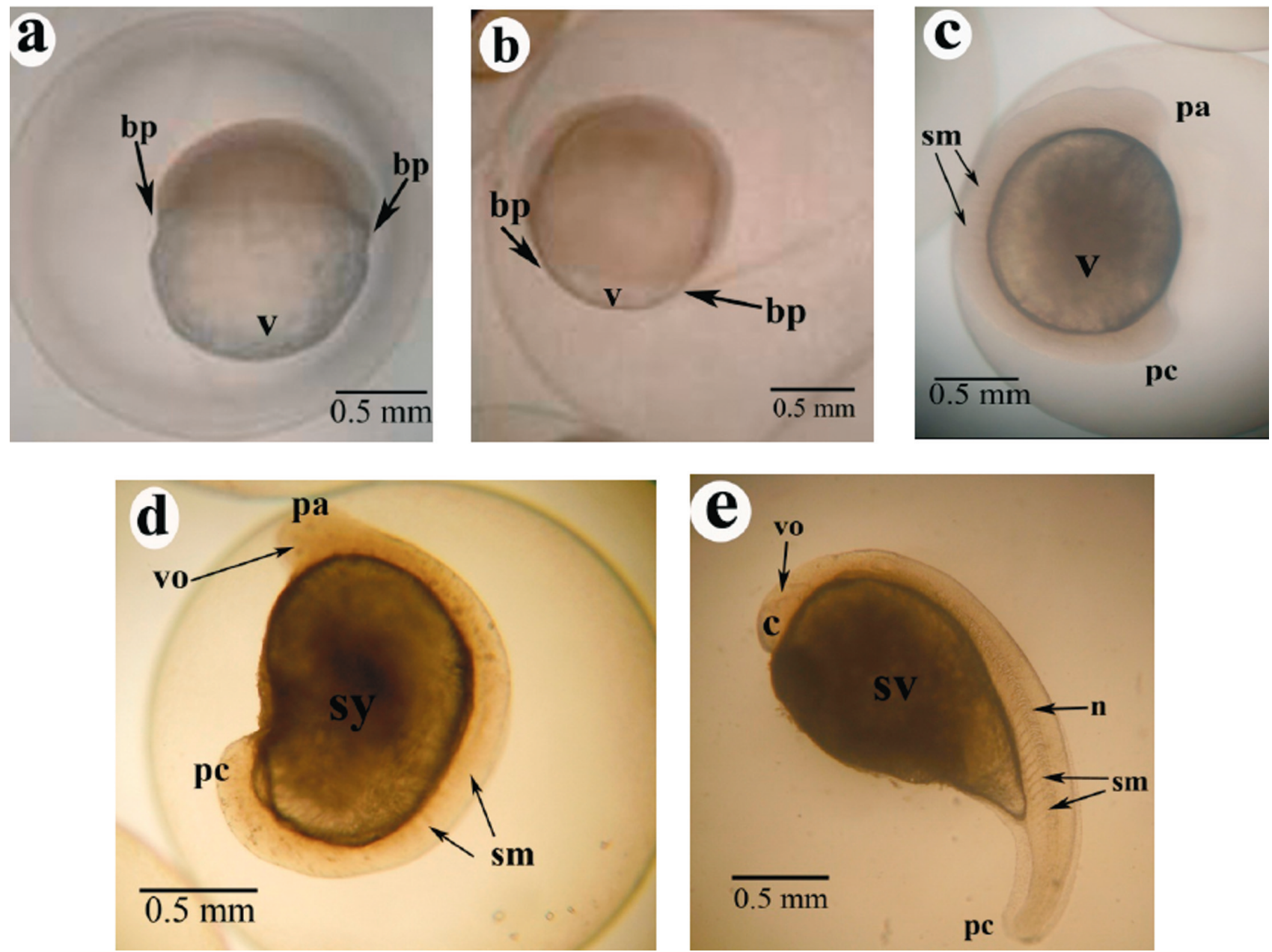

Fig. 4. Formación de la gástrula y organogénesis en Piaractus brachypomus. a) epibólia del 50\%, b) epibólia del 80 \%, c-d) embrión diferenciado antero-posterior en forma arqueada, e) larva eclosionada. (bp) borde periblasto, (v) vitelo, (tv) tapón vitelino, (sm) somitas, (vo) vesícula óptica, (n) notocorda, (pa) parte anterior, (pc) parte caudal, (sv) saco vitelino.

formación del cristalino y los otolitos. Durante la última fase (organogénesis iv o tardía) se observaron somitas pareadas, vasos del sistema circulatorio y formación de aletas primigenias. Este proceso en Pseudoplatystoma $s p$ se inició cerca de las 8:00 HPF y se extendió hasta las 16:00 HPF, mientras que en $P$. brachypomus se observó desde las 8:00 hasta 13:00 HPF.

Eclosión. La eclosión en Pseudoplatystoma sp se observó desde las 14:00 HPF hasta las 18:00 HPF, mientras que en $P$. Brachypomus se inició a las 12:00 HPF y llego al 80\% de larvas eclosionadas a las 14:00 HPF (Fig.6).

\section{DISCUSIÓN}

Los oocitos de Pseudoplatystoma sp tienen una doble membrana característica de los siluriformes (Cardoso et al.; Sanches et al., 1999; Núñez et al.; Marques et al.), mientras que P. brachypomus poseen una única membrana (Botero et al.). Sin embargo, una de las diferencias más importantes entre las dos especies son las membranas que recubren el cigoto y el tamaño del espacio perivitelínico, así: en Pseudoplatystoma $s p$ existe un mayor espacio que en $P$. brachypomus; de igual forma, el diámetro de los oocitos hidratados después de la fertilización de Pseudoplatystoma $s p$ es mayor $(1930 \pm 40 \mu \mathrm{m})$ que los de P. brachypomus (1680 $\pm 34 \mu \mathrm{m}$ ), lo cual le proporcionaría al embrión una mayor protección de los daños mecánicos del ambiente (Cardoso et al.; Pereira et al.). Ha sido observado también que especies que tienen un espacio perivitelino reducido como Rhamdia hilarii, sus desoves ocurren en aguas poco turbulentas y de baja corriente (Godinho et al.; Pereira et al.).

Las dos especies presentaron un patrón de clivaje meroblástico, que ocurre en el polo animal, lo cual es típico de los oocitos de peces con gran cantidad de vitelo o 


\section{P. fasciatum}
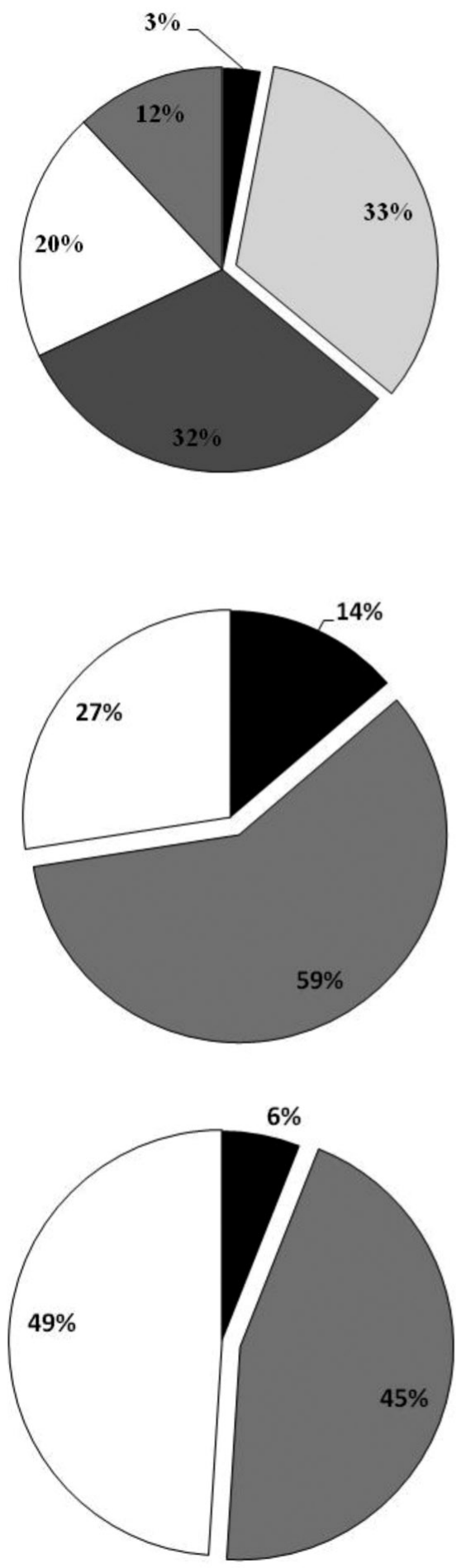

P. brachypomus

- 4 Células

$\square 8$ Células

$\square 16$ Células

$\square 32$ Células

๑64 Células

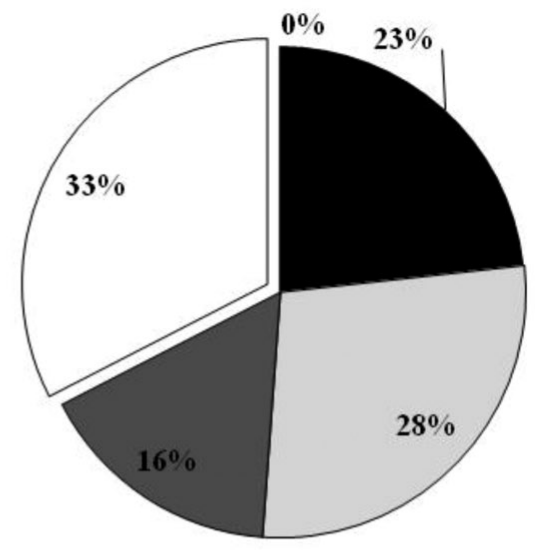

- Blástula inicial

口Elevación

Blastodisco

$\square$ Descenso

Blastodisco

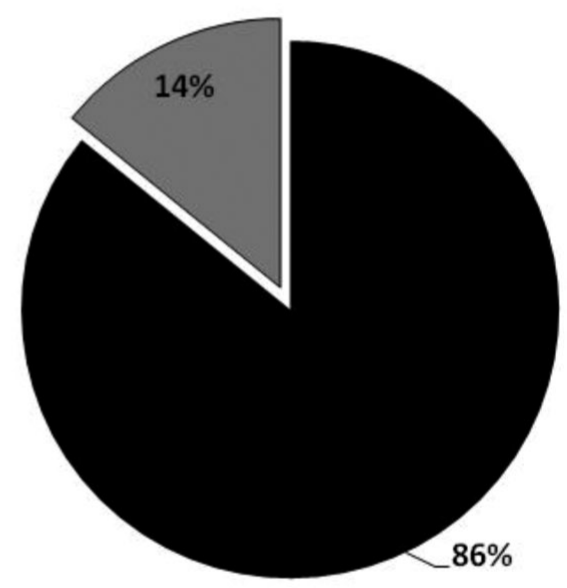

-Elevación

Blastodisco

$\square$ Descenso

Blastodisco

$\square$ Inicio

Gastrulación

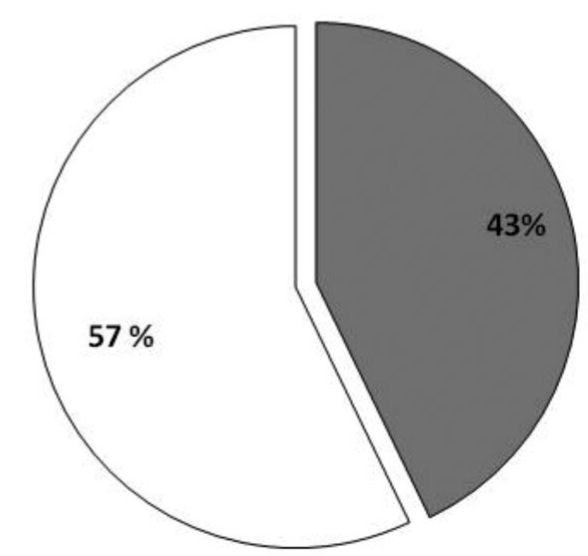

Fig. 5. Porcentaje relativo de los estadios del desarrollo embrionario en Pseudoplatystoma sp y P.brachypomus desde la primera hora post fertilización (HPF) hasta la $5 \mathrm{~h}$. 


\section{P. fasciatum}
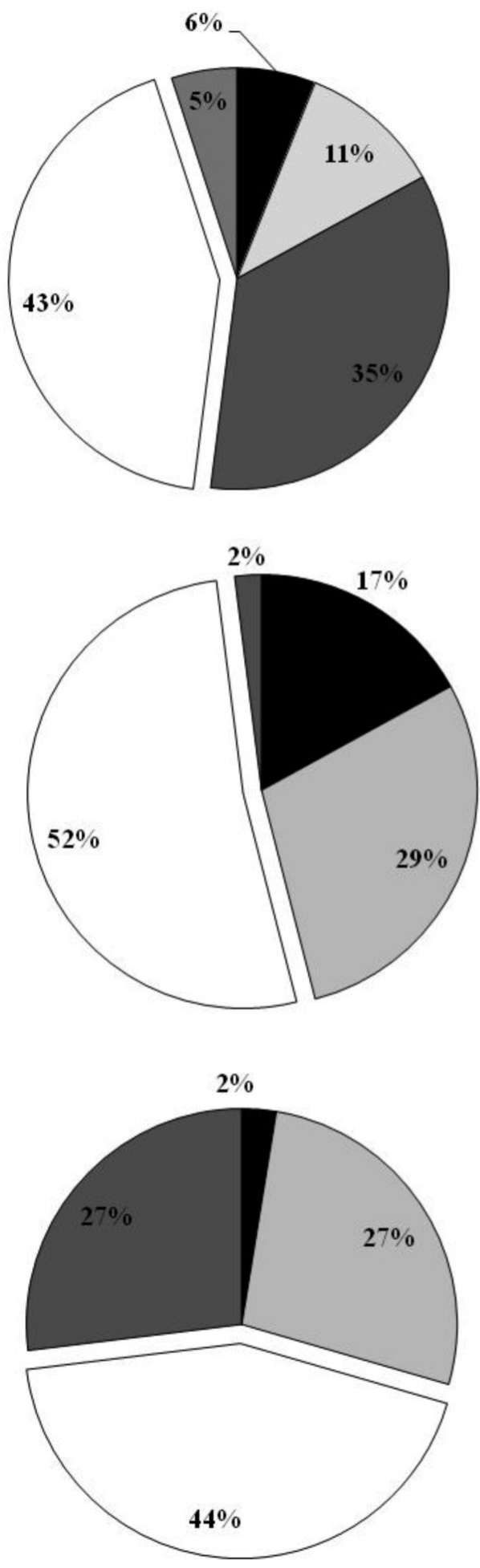

\section{P. brachypomus}

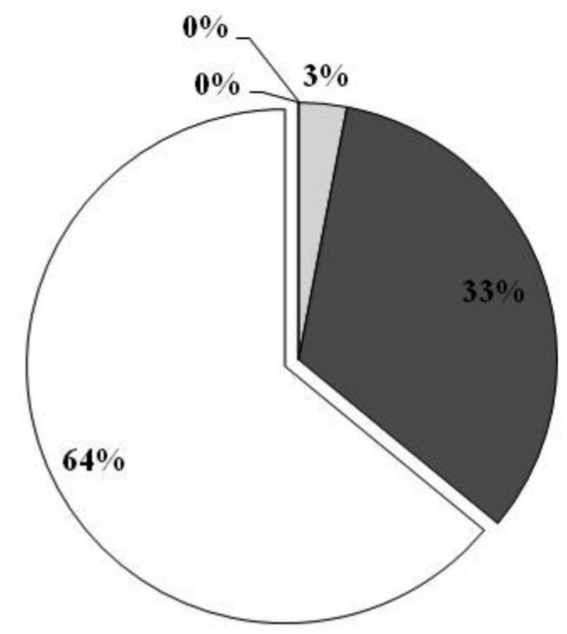

Inicio

Gastrulación

$\square$ Epibolia $50 \%$

Epibolia 80-

$90 \%$

Cierre

Blastoporo
- Cierre Blastoporo

$\square$ Organogénesis i

$\square$ Organogénesis ii

$\square$ Organogénesis iii

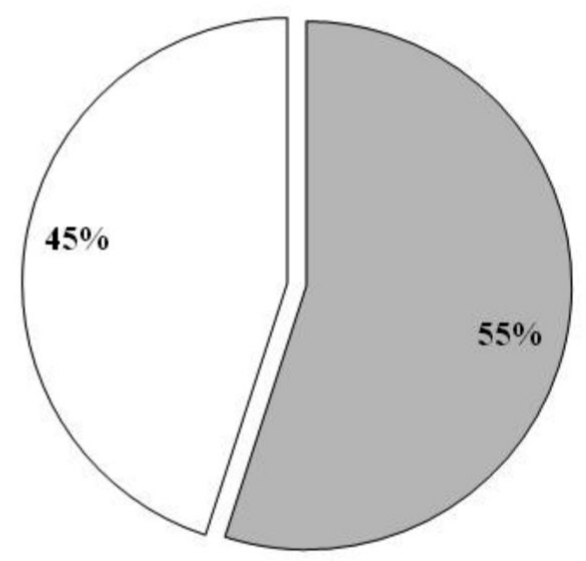

- Organogénesis ii

$\square$ Organogénesis iii

$\square$ Organogénesis iv

\section{Eclosión}

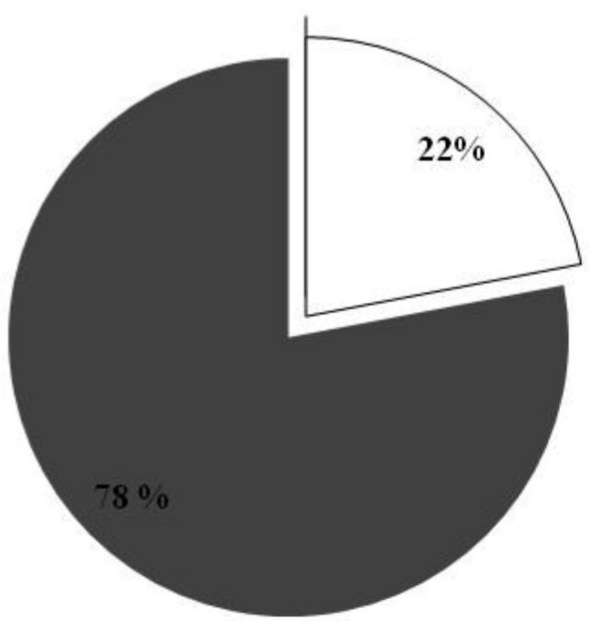

Fig. 6. Porcentaje relativo de los estadios del desarrollo embrionario en Pseudoplatystoma sp y P. brachypomus desde la $8 \mathrm{~h}$ post fertilización (HPF) hasta las $14 \mathrm{~h}$. 
telolecitos (Cardoso et al.; Botero et al.; Marques et al.; Ninhaus-Silveira et al.). Los primeros clivajes observados en las dos especies estudiadas siguen el mismo patrón de segmentación que los reportados para otros silúridos tales como Rhamdia quelen, Rhamdia sapo, Rhamdia hilarii, Pseudoplatystoma corruscans (Pereira et al.; Cardoso et al.; Cussac et al.; Marques et al.), así como para otras especies de characiformes como el híbrido de Piaractus brachypomus por Colossoma macropomum (Botero et al.). El tiempo transcurrido desde la fertilización hasta llegar al quinto (32 células) y sexto clivajes (64 células) fluctuó entre 1:00 y 2:00 HPF para Pseudoplatystoma sp (Fig. 1b-e) y entre 1:00 -1:30 HPF para $P$. brachypomus $(2 \mathrm{~b}-\mathrm{g})$, a una temperatura entre 26.9 - $27.1^{\circ} \mathrm{C}$. A esta temperatura el Pseudoplatystoma sp presenta un ritmo más heterogéneo en la división (Fig. 5), mientras que $P$. brachypomus es más rápido y más homogéneo con respecto a Pseudoplatystoma sp bajo las mismas condiciones. En otras especies reportadas de siluriformes como Rhamdia quelen el estado de 128 células se observó a las cuatro HPF a una temperatura $24 \pm 1^{\circ} \mathrm{C}$ (Pereira et al.), mientras que en Pseudoplatystoma coruscans se observó a la 1:30 $\mathrm{HPF} \mathrm{a} 28^{\circ} \mathrm{C}$ (Marques et al.); de igual forma, en characiformes como Prochilodus lineatus, se observó a las 2:25 HPF a $24^{\circ} \mathrm{C}$ o 1:34 HPF a $28^{\circ} \mathrm{C}$ (Ninhaus-Silveira et al.); en el hibrido de Piaractus brachypomus por Colossoma macropomum se observó a las 1:45 $\mathrm{HPF}$ a $27^{\circ} \mathrm{C}$ (Botero et al.).

El paso de blástula a gástrula, conocido como el proceso de epibolia, alcanzó el 50\%, luego llega al 80-95\% hasta llegar al cierre del blastoporo, donde finalmente el epiblasto forma el ectodermo y el hipoblasto forma la notocorda y el mesodermo (Adriaens \& Vandewalle, 2003); en Pseudoplatystoma sp el cierre del blastoporo ocurrió entre las 7:00 y 12:00 HPF (Fig. 3c), mientras que en $P$. brachypomus ocurrió entre las 7:00 y 10:00 HPF (Fig. 4b), siendo más corto y homogéneo que en Pseudoplatystoma sp (Fig. 6). En silúridos, como Rhamdia quelen, se ha reportado el cierre del blastoporo a las 10:30 HPF (Pereira et al.) y en Pseudoplatystoma corruscans a las 6:00 HPF (Cardoso et al.; Marques et al.) y Rhamdia hillarii (Godinho et al.). En carácidos como Prochilodus lineatus (Ninhaus-Silveira et al.) se observó el cierre del blastoporo alrededor de las 8:00 HPF y en el hibrido de Piaractus brachypomus por Colossoma macropomum a las 10:30 HPF (Botero et al.).

En Pseudoplatystoma sp antes de la eclosión se observaron aproximadamente 25 somitas, mientras que en $P$. brachypomus alrededor de 14 . Este proceso en Pseudoplatystoma sp transcurrió entre la 8:00 HPF y 16:00 HPF, mientras que en $P$. brachypomus fue más homogéneo, observándose entre las 8:00 y 13:00 HPF.

La duración de la organogénesis fue menor en $P$. brachypomus con respecto a Pseudoplatystoma sp, pero se observó un menor desarrollo morfológico de estructuras tales como las cavidades cefálicas y menor número de somitas diferenciadas. En silúridos, tal como Pseudoplastystoma corruscans, este proceso se observó entre las 7:30 y 14:30 HPF (Cardoso et al.), en Rhamdia quelen entre las 11:00 y 19:00 HPF (Pereira et al.), en Rhamdia hilarii entre las 7:30 y 9:00 HPF (Godinho et al.) y en Carácidos como Prochilodus lineatus, la organogénesis se observó entre las 10:00 y 16:00 HPF (Ninhaus-Silveira et al.), muy similar a lo reportado por Botero et al. para el hibrido de Piaractus brachypomus por Colossoma macropomum (10:00 y 17:00 HPF).

En Pseudoplatystoma sp, la eclosión comenzó a partir de las 14:00 HPF y duró hasta las 18:00 HPF, mientras que en $P$. Brachypomus se inició a las 12:00 HPF y terminó hacia las 14:00 HPF, cuando el 80\% de larvas habían eclosionado (Fig.6). Esta diferencia en el momento de inicio y duración de la eclosión, puede explicar la mayor asincronía en el desarrollo larvario observada en Pseudoplatystoma sp, permitiéndole a esta especie que un mayor número de larvas alcancen su desarrollo cefálico y abran la boca más temprano, incurriendo en canibalismo cuando se termina la alimentación endógena y pasa al tipo de alimentación exógena.

Así la heterogeneidad en el desarrollo embrionario y larval es un problema central en la larvicultura, especialmente en especies predadoras como Pseudoplatystoma sp. Estas diferencias morfológicas que contribuyen a la heterogeneidad en el tamaño pueden ser atribuidas a diferencias en los tiempos la eclosión; en Pseudoplatystoma sp este proceso se observó desde las 14:00 HPF y se extendió hasta las 18:00 HPF, dando origen a un crecimiento asincrónico que permitió que las primeras larvas desarrollen rápidamente su boca y por tanto la relación boca-tamaño del cuerpo les proporciona mayores habilidades competitivas al momento de consumir larvas hermanas ligeramente más pequeñas (Baras \& Jobling, 2002; Kestemont et al., 2003).

Sin embargo, el poco conocimiento sobre el desarrollo embrionario y larval de estas especies de interés comercial, dificulta el desarrollo de los procesos productivos durante la larvicultura y alevinaje, siendo necesarios trabajos adicionales que aporten conocimiento a la biología de estas especies y permitan establecer técnicas eficientes para su producción con fines de piscicultura.

\section{AGRADECIMIENTOS}

Al Instituto de Acuicultura de la Universidad de los Llanos (IALL - UNILLANOS), por la colaboración en la fase de campo para la realización de este proyecto. 
DÍAZ-OLARTE, J.; MARCIALES-CARO L. J.; CRISTANCHO, V. F. \& CRUZ-CASALLAS, P. E. Comparison of the embryonic development of Piaractus brachypomus (Serrasalmidae) and Pseudoplatystoma sp. (Pimelodidae). Int. J. Morphol., 28(4):1193-1204, 2010 .

SUMMARY: Pseudoplatystoma sp. and Piaractus brachypomus are two species of freshwater fishes endemic to South America as a high commercial crop species. The aim of this study was to compare the embryonic development of these two species until they hatch. To observe embryonic development fertilized oocytes were incubated obtained by hormonal induction in upwelling incubators Woynarovich type under controlled conditions of water quality. The cleavages were observed for Pseudoplatystoma sp. from 0:30 hours post fertilization (HPF) until 2:00 pm (HPF), whereas in P. brachypomus these occurred between 0:30 - 1:30 HPF. The stage of blastula, gastrula until the closure of blastopore Pseudoplatystoma sp. was observed in sp between 2:00 to 12:00 P. HPF while in P. brachypomus between 2:00 to 8:00. The hatching in Pseudoplatystoma sp was observed between 14:00 to 18:00 HPF, whereas P. brachypomus was faster, was observed from 12:00 until 14:00 HPF HPF, and was more homogeneous than Pseudoplatystoma $\mathrm{sp.}$

KEYWORDS: Embryonic development; P. Brachypomus; Pseudoplatystoma sp.

\section{REFERENCIAS BIBLIOGRÁFICAS}

Adriaens, D. \& Vandewalle P. Embryonic and larval development in catfishes. In: Arratia, G.; Kapoor, B. G.; Diogo, R. \& Chardon, M. Catfishes. Vol. 2. New Hampshire, Sciences Publisher Inc., 2003. pp.639-68.

Atencio-García, V. \& Zaniboni-Filho, E. El canibalismo en la larvicultura de peces. Rev. MVZ Córdoba, 11:9-16, 2006.

Baras, E. \& Jobling, M. Dynamics of intracohort cannibalism in cultured fish. Aquacult. Res., 33:461-79, 2002.

Botero, M.; Fresnada, A.; Montoya, A. \& Olivera, M. Descripción del desarrollo embrionario de zigotos híbridos obtenidos por el cruce de machos de Cachama Blanca (Piaractus brachypomus) y hembras de Cachama Negra (Colossoma macropomum). Rev. Col. Cienc. Pec., 17:38-45, 2004.

Cardoso, E. L.; Alves, M. S.; Ferreira, R. M. \& Godinho, H. P. Embryogenesis of the neotropical freshwater Siluriforme Pseudoplatystoma coruscans. Aquat. Living Resour., 8:343-6, 1995.

Cussac, V.; Matkovic, M. \& Maggese, M. Desarrollo embrionario de Rhamdia sapo (Valenciennes, 1840) Eigenmann y Eigenmann; 1888 (Pisces, Pimelodidae) II. Organogénesis media, Organogénesis tardía y eclosión. Rev. Brasil. Biol., 45:149-60, 1985.

Fabré, N. N.; Donato, J. C. \& Alonso, J. C. Bagres de la Amazonia Colombiana: Un Recurso Sin Fronteras. Bogotá, Instituto Amazónico de Investigaciones Científicas Sinchi. Programa de Ecosistemas Acuáticos, 2000. pp.19-70.
Faustino, F.; Okada, L.; Marques, C.; Makino, L. \& Senhorini, J. A. Fertilização e desenvolvimento embrionário: morfometria e análise estereomicroscópica dos ovos híbridos de surubins (pintado, Pseudoplatystoma corruscans $\mathrm{x}$ cachara, Pseudoplatystoma fasciatum). Acta Sci. Biol. Sci. Maringá, 29:49-55, 2007.

Godinho, H.; Fenerich, N. \& Narahara, M. Desenvolvimento embrionário e larval de Rhamdia hilarii (Valenciennes, 1840) (Siluriformes, Pimelodidae). Rev. Brasil. Biol, 38:151-6, 1978.

Kestemont, P.; Jourdan, S.; Houbart, C.; Paspatis, M.: Fontaine, P.; Cuvier, A. et al. Size heterogeneity, cannibalism and competition in cultured predatory fish larvae: biotic and abiotic influences. Aquaculture, 227:333-56, 2003.

Kossowski, C. Perspective de L'élevage des poisons-chats (Siluroidei) en Amérique du Sud. Aquat. Living Resour, Hors Série:189-95, 1996.

Landines, M. A. \& Mojica, H. O. Manejo y reproducción de Carácidos. En: Daza, P.; Landines, M. \& Sanabria, A. (Eds.). Reproducción de Peces en el Trópico, Vol. 1. Bogotá, Ed. Imprenta Nacional de Colombia, 2006. pp.91-104.

Machinandiarena, L.; Müller, M. \& López, A. Early life of development of the red porgy Pagrus pagrus (Pisces, Sparidae) in captivity, Argentina. Invest. Mar. Valparaiso, 3:5-13, 2003.

Marques, C.; Okada, L.; Faustino, F.; Nakaghi, L. \& 
DÍAZ-OlARTE, J.; MARCIALES-CARO L. J.; CRISTANCHO, V. F. \& CRUZ-CASALlAS, P. E. Comparación del desarrollo embrionario de Piaractus brachypomus (Serrasalmidae) y Pseudoplatystoma sp. (Pimelodidae). Int. J. Morphol., 28(4):1193-1204, 2010.

Senhorini, J. Observation of the embryonic development in Pseudoplatystoma coruscans (Siluriformes: Pimelodidae) under light and scanning electron microscopy. Zygote, 16:333-42, 2008.

Mira, T.; Castro, S.; Medina, V.; Murillo, R.; Otero, A.; Ramírez, J.; et al. Ensayos preliminares de reproducción inducida de bagre rayado Pseudoplatystoma fasciatum con extracto de hipófisis de carpa. En memorias de la XIII Jornada de Acuicultura Unillanos, Villavicencio, Meta, 2007. pp.65-7.

Ninhaus-Silveira, A.; Foresti, F. \& de Azevedo, A. Structural and ultrastructural analysis of embryonic development of Prochilodus lineatus (Valenciennes, 1836) (Characiforme; Prochilodontidae). Zygote, 14:217-29, 2006.

Nuñez, J.; Duqué, R.; Corcuy-Arana, N.; Duponchelle, F.; Renno, J. F.; Raynaud, T.; et al. Induced breeding and larval rearing of Surubí, Pseudoplatystoma fasciatum (Linnaeus, 1766), from the Bolivian Amazon. Aquac. Res., 39:764-76, 2008.

Pereira, C. R.; Barcellos, L.; Kreutz, L. C.; Quevedo, R. M.; Ritter, F. \& Silva. Embryonic and larval development of jundiá (Rhamdia quelen, Quay \& Gaimard, 1824, pisces, Teleostei), a South American catfish. Braz. J. Biol., 66:1057-63, 2006.

Qin, J. \& Fast, A. Size and feed dependent cannibalism with juvenile snakehead Chana striatus. Aquaculture, 144:313-20, 1996.

Sanches, P. V.; Nakatani, K. \& Bialetzki, A. Morphological description of the developmental stages of Parauchenipterus galeatus (Linnaeus, 1766) (Siluriformes, Auchenipteridae) on the floodplain of the upper Paraná river. Rev. Bras. Biol., 59:429-38, 1999.

Segura, L.; Hayashi, C.; de Souza, S. \& Soares, C. Canibalismo entre larvas de pintado, Pseudoplatystoma corruscans, cultivadas sob diferentes densidades de estocagem. A. Scient. Biol. Sci., 26:299-302, 2004.

Zaniboni-Filho, E.; Kennedy, L. \& Reynalte-Tataje, A. Desenvolvimento embrionário e estágios larvais do mandi-amarello Pimelodus maculatus. B. Inst. Pesca, 27:49-55, 2001.
Dirección para correspondencia:

Lili J. Marciales-Caro

Instituto de Acuicultura

Universidad de los Llanos

Km 12 vía Puerto López

Villavicencio - Meta

COLOMBIA

Email: lilimarciales@gmail.com

Recibido : 27-08-2010

Aceptado: 29-09-2010 